\title{
Drug resistance of cancer cells is crucially affected by expression levels of $A B C$-transporters
}

\author{
Petr Mlejnek ${ }^{\ddagger}$, Petr Dolezel ${ }^{\ddagger}$, Eliska Ruzickova ${ }^{\ddagger}$ \\ ‡ Palacky University Olomouc, Olomouc, Czech Republic
}

Corresponding author: Petr Mlejnek (mlejnek petr@volny.cz)

Academic editor: Nikolai Zhelev

Received: 15 Nov 2016 | Accepted: 24 Jan 2017| Published: 08 Feb 2017

Citation: Mlejnek P, Dolezel P, Ruzickova E (2017) Drug resistance of cancer cells is crucially affected by expression levels of ABC-transporters. BioDiscovery 20: e11211. https://doi.org/10.3897/biodiscovery.20.e11211

\begin{abstract}
Dasatinib (DAS), a second generation of tyrosine kinase inhibitor (TKI), represents excellent choice for the treatment of chronic myeloid leukemia resistant to imatinib. Unfortunately, recent laboratory studies suggested that antiproliferative effect of DAS might be significantly reduced due to the overexpression of the ATP-binding cassette (ABC) transporters, $A B C B 1$ and $A B C G 2$. However, whether these drug transporters might compromise therapeutic effect of DAS in clinic is unclear. We believe that the drug transporter expression level is a crucial factor that affects the results and its consideration may help to explain the existing controversy. In addition, clinically relevant concentrations of drug must be used.
\end{abstract}

In our study, human leukemia K562 cells with high and low expression levels of ABCB1 or ABCG2 were used. DAS was applied at nanomolar concentrations. We observed that K562 cells expressing high levels of ABCB1 and ABCG2 contained significantly reduced intracellular levels of DAS and these cells exhibited significantly increased resistance to this drug. Importantly, cells with the low expression of ABCB1 or ABCG2 effluxed DAS less efficiently, however, still significantly. Accordingly, the observed resistance was lower but significant. Conclusions: The antiproliferative effects of DAS might be reduced by $A B C B 1$ or ABCG2. However, the actual effect of these ABC transporters on DAS efficiency depends on their expression levels. The lower expression levels of $A B C$ transporters mediate lower resistance. Considering the fact that expression levels of $A B C B 1$ and 
ABCG2 transporters are usually low in clinical samples, their contribution to the overall resistance to DAS is probably low but significant.

\section{Keywords}

Dasatinib, ABCB1, ABCG2, transporter expression level, drug resistance

\section{Introduction}

A detailed understanding of the molecular cause of cancer enabled "targeted" treatment: an effective treatment with a minimal damage for healthy cells. Targeted therapy utilises rationally designed drugs that interfere with specific molecules (molecular targets) essential for proliferation and survival of malignant cells. Targeted therapy brought about a revolution in cancer treatment in the last decades (Dy and Adjei 2008).

Tyrosine kinases are especially important target because they play a crucial role in the modulation of number specific signal transduction pathways including cell proliferation and differentiation and therefore if mutated they have the potential to induce oncogenic transformation. Tyrosine kinase inhibitors (TKIs), low molecular weight inhibitors that compete with the ATP binding site of the catalytic domain of oncogenic tyrosine kinases, exhibit effective antitumor activity both in vitro and in vivo. They represent an example of successful targeted therapy (Dy and Adjei 2008, Kantarjian et al. 2007).

Despite the promising results of TKIs in clinical trials and clinical practise, recent in vitro studies suggested that their efficiency might be compromised due to the overexpression of the ATP-binding cassette (ABC) transporters, namely ABCB1 (P-glycoprotein, MDR1) and ABCG2 (breast cancer resistance protein, BCRP). However, the possible involvement of $A B C$ transporters in clinical multidrug resistance is difficult to establish. Indeed, the data obtained from in vitro experiments convincingly suggest that particular members of $A B C$ transporter family can mediate multidrug resistance (MDR) in cancer cells. Based on the laboratory findings it was suggested that inhibition of the $A B C$ drug transporter could sensitize drug-resistant tumours. Although this hypothesis is rational, the clinical trials evaluating this strategy mostly have failed to reach a positive endpoint (Tamaki et al. 2011, Amiri-Kordestani et al. 2012). Explanation of the existing controversy between laboratory and clinical results is difficult as many factors may have an impact on the results. We believe that the drug transporter expression level is a crucial but often overlooked factor that affects the results and thus may help to explain existing controversy.

Here, we addressed the question how expression levels of ABCB1 and/or ABCG2 affect the cell resistance to DAS. We observed that the expression level of the studied ABCtransporter is an important factor that affects the cell resistance. While cells expressing high levels of drug transporters, which are often used in laboratory experiments, exhibit a high degree of resistance to DAS, cells with low expression levels of drug transporters, 
which can occur in clinical samples, exhibit much lower but significant degree of resistance to DAS.

\section{Results}

\section{Isolation and characterization of cells expressing ABCB1 and ABCG2}

In our study, human leukemia cells with high and low expression levels of $A B C B 1$ or ABCG2 were used. Expression levels of the ABCB1 transporter in K562/Dox and K562/ DoxDR1 cells are given in Fig. 1a, b. Cells overexpressing ABCG2, K562/ABCGCL10 and K562/ABCGCL1, are characterized in Fig. 1c, d. Parental K562 cells, which do not express either of the transporters, were used as a control. Analysis of ABCB1 and ABCG2 function was done using the calcein accumulation assay (Holló et al. 1994) and pheophorbide $A$ accumulation assay, respectively (data not shown; Robey et al. 2004). Detailed characterization of these cells is given elsewhere (Kosztyu et al. 2014).

\section{The effect of ABCB1 and ABCG2 expression on intracellular levels of dasatinib}

The reduction of intracellular drug concentrations which prevents accessibility of drugs to their sites of action is a generally accepted mechanism of resistance mediated by $A B C$ transporters (Gottesman 1993, Ambudkar et al. 2003). Therefore, we measured the effect of $A B C B 1$ and $A B C G 2$ expression on DAS intracellular levels. Experiments were conducted for 300nM DAS, a concentration which is relevant to that used in clinics (Eadie et al. 2014). We observed that K562/Dox and K562/ABCGCL10 cells with high expression of $A B C B 1$ and $A B C G 2$, respectively, contained significantly reduced intracellular levels of DAS (Fig. 2). Importantly, K562/DoxDR1 and K562/ABCGCL1 cells with the low expression of $A B C B 1$ and $A B C G 2$, respectively, effluxed DAS with lower efficiency than cells with high transporter expression levels (Fig. 2). However, even cells with the low expression of ABCB1 or ABCG2 reduced intracellular level of DAS significantly (Fig. 2).

\section{The effect of dasatinib on cell viability and apoptosis induction in cells expressing $A B C B 1$ or $A B C G 2$}

Owing to the fact that DAS inhibits cell proliferation and induces apoptosis (Nam et al. 2007), cell sensitivity to this drug was measured using the standard MTT assay and by analysis of morphological and biochemical apoptotic hallmarks. In agreement with the previous results, K562/Dox and K562/ABCGCL10 cells with high expression of ABCB1 and ABCG2, respectively, exhibited an increased resistance to DAS (Fig. 3a, b and Table 1). Accordingly, K562/DoxDR1 and K562/ABCGCL1 cells with the low expression of ABCB1 and $A B C G 2$, respectively, exhibited decreased but significant resistance to DAS (Fig. 3c and Table 1). The observed resistance was counteracted by ZSQ, a specific inhibitor of ABCB1, and by Ko143, a specific inhibitor of ABCG2 (Fig. 3, Table 1). Exactly the same cell sensitivity pattern in cells expressing high and low levels of ABCB1 or ABCG2 was obtained using flow cytometric analysis of hypodiploid (apoptotic) cells (not shown). 


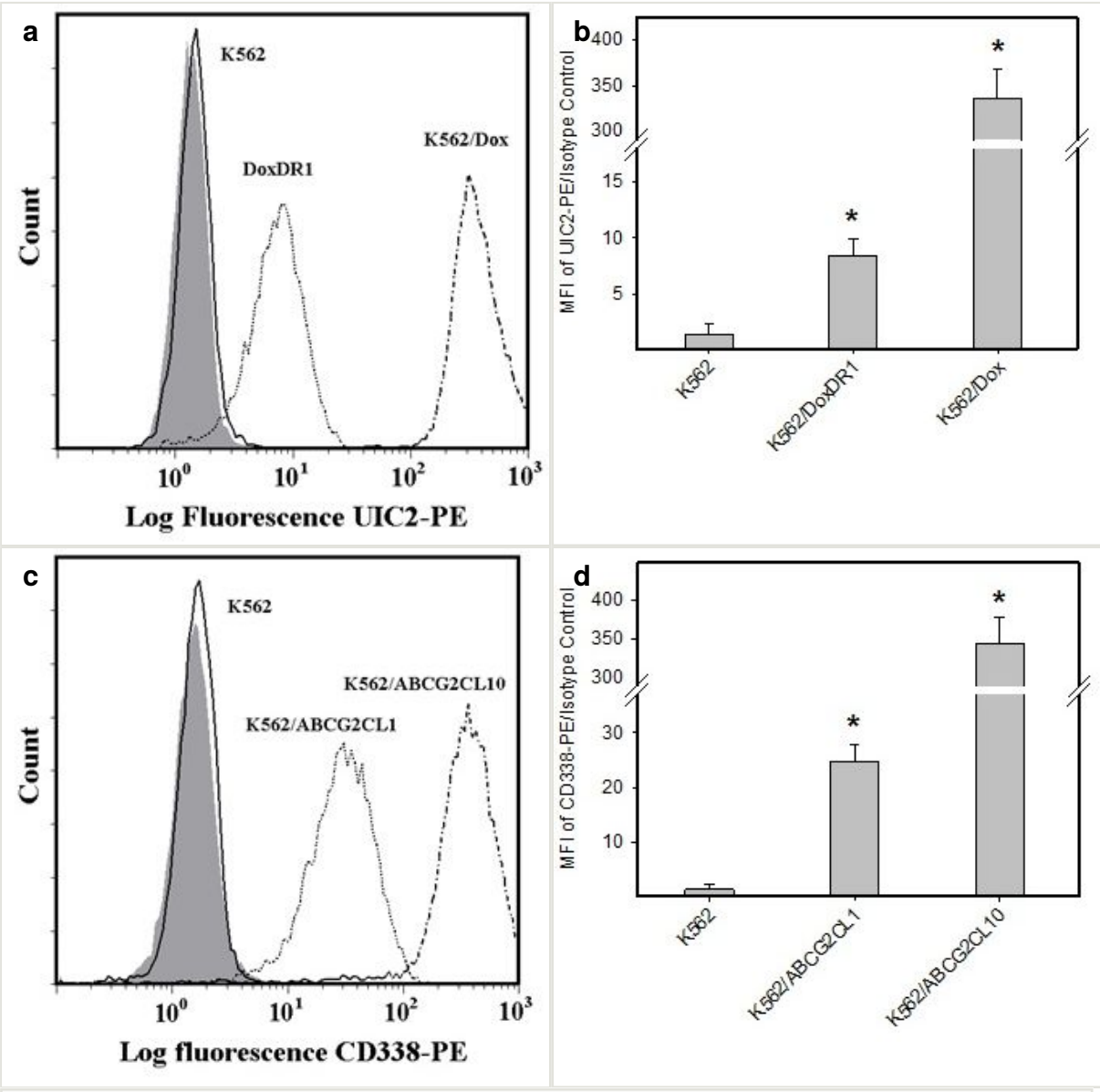

Figure 1.

Analysis of $A B C B 1$ and $A B C G 2$ expression.

a: Flow cytometric analysis of ABCB1 expression. Isotype control (grey histogram); K562 parental cell line (solid line); K562/Dox cells (dash-dot line); K562/DoxDR1 cells (dot line).

b: Quantitative analysis of $A B C B 1$ expression. $A B C B 1$ expression was quantified as the mean fluorescence intensity (MFI) shift (ratio of MFI of UIC2-PE antibody and isotype control). The experimental points represent mean values from three replicate experiments, with standard deviations. * denotes significant change in $A B C B 1$ expression $(P<0.05)$ between $\mathrm{K} 562$ cells and cells expressing various levels of ABCB1 (K562/Dox, K562/DoxDR1).

c: Flow cytometric analysis of ABCG2 expression. Isotype control (grey histogram); K562 parental cell line (solid line); K562/ABCG2CL10 cells (dash-dot line); K562/ABCGCL1 cells (dot line).

d: Quantitative analysis of $A B C G 2$ expression. ABCG2 expression was quantified as the mean fluorescence intensity (MFI) shift (ratio of MFI of CD338-PE antibody and isotype control). The experimental points represent mean values from three replicate experiments, with standard deviations. * denotes significant change in ABCG2 expression $(P<0.05)$ between $K 562$ cells and cells expressing various levels of ABCG2 (K562/ABCGCL10, K562/ABCGCL1). 

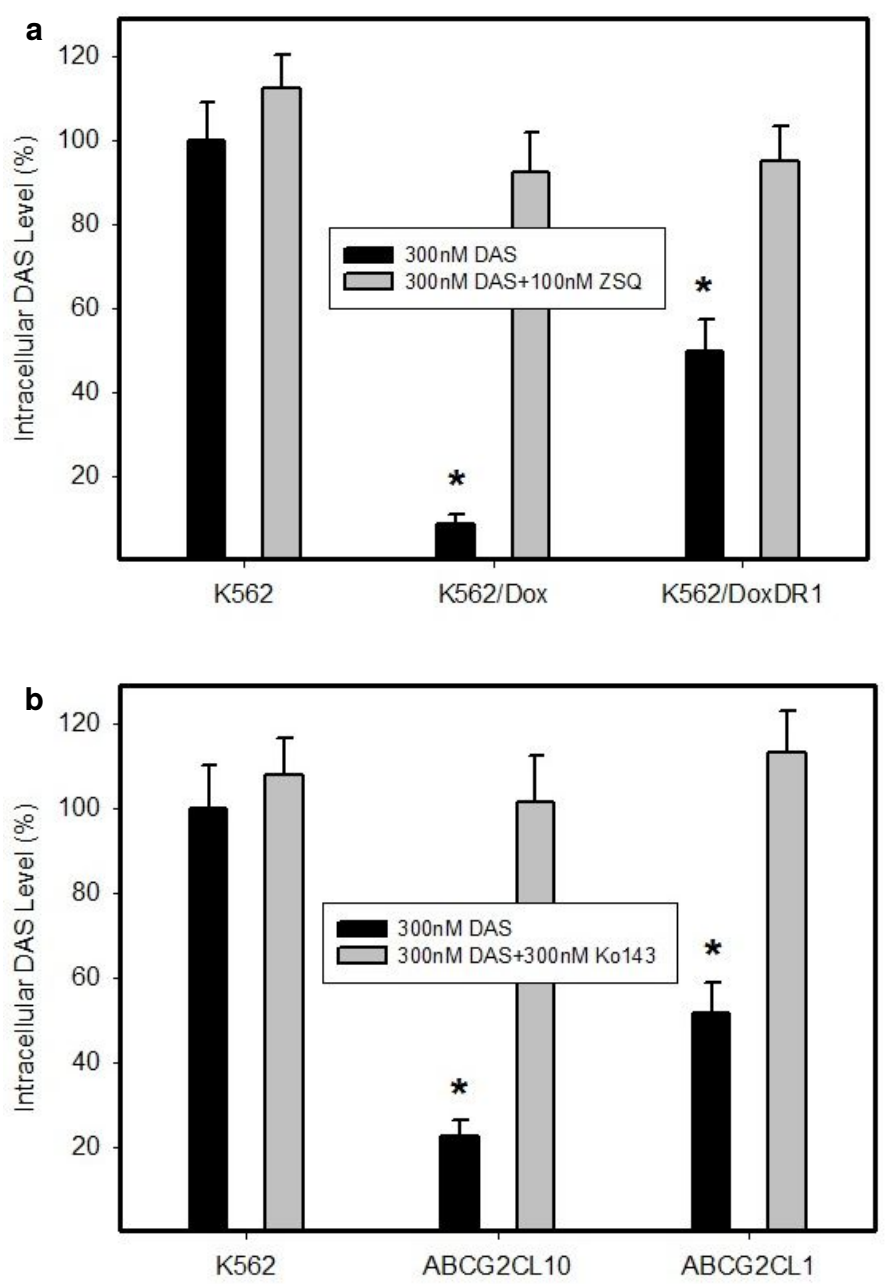

Figure 2.

Intracellular levels of DAS in cells expressing ABCB1 or ABCG2. Parental cell line K562, which does not express any of transporter was used as a control. Cells were incubated with $300 \mathrm{nM}$ DAS or with 300nM DAS + appropriate inhibitor (100nM ZSQ or 300nM Ko143), as indicated. Intracellular level of DAS was determined after $3 \mathrm{~h}$ incubation at $37^{\circ} \mathrm{C}$. The experimental points represent mean values from three replicate experiments, with standard deviations.

a: A relative intracellular levels of DAS in cells overexpressing $A B C B 1$ transporter. * denotes significant change in intracellular level of DAS $(P<0.05)$ between $K 562$ cells and cells expressing various levels of ABCB1, K562/Dox and K562/DoxDR1.

b: A relative intracellular levels of DAS in cells overexpressing ABCG2 transporter. * denotes significant change in intracellular level of DAS $(P<0.05)$ between $\mathrm{K} 562$ cells and cells expressing various levels of ABCG2, K562/ABCG2CL10 and K562/ABCG2CL1. 
Table 1.

Effect of DAS on cell survival and viability in cells with high and low expression levels of $A B C B 1$ and ABCG2. Cells were treated with DAS for $48 \mathrm{~h}$ prior to MTT assay.

\begin{tabular}{|c|c|c|}
\hline Cell line & IC $_{\mathbf{5 0}}$ for DAS & IC $_{\mathbf{5 0}}$ for DAS+Inhibitor \\
\hline K562 & $0,38 \pm 0,13(\mathrm{nM})$ & $0,43 \pm 0,15(\mathrm{nM})$ \\
\hline K562/Dox & $7,66 \pm 0,84(\mathrm{nM})$ & $0,41 \pm 0,21(\mathrm{nM})$ \\
\hline K562/DoxDR1 & $0,82 \pm 0,17(\mathrm{nM})$ & $0,45 \pm 0,14(\mathrm{nM})$ \\
\hline K562/ABCG2CL10 & $5,34 \pm 0,71(\mathrm{nM})$ & $0,54 \pm 0,23(\mathrm{nM})$ \\
\hline K562/ABCG2CL1 & $0,89 \pm 0,18(n M)$ & $0,52 \pm 0,14(\mathrm{nM})$ \\
\hline
\end{tabular}
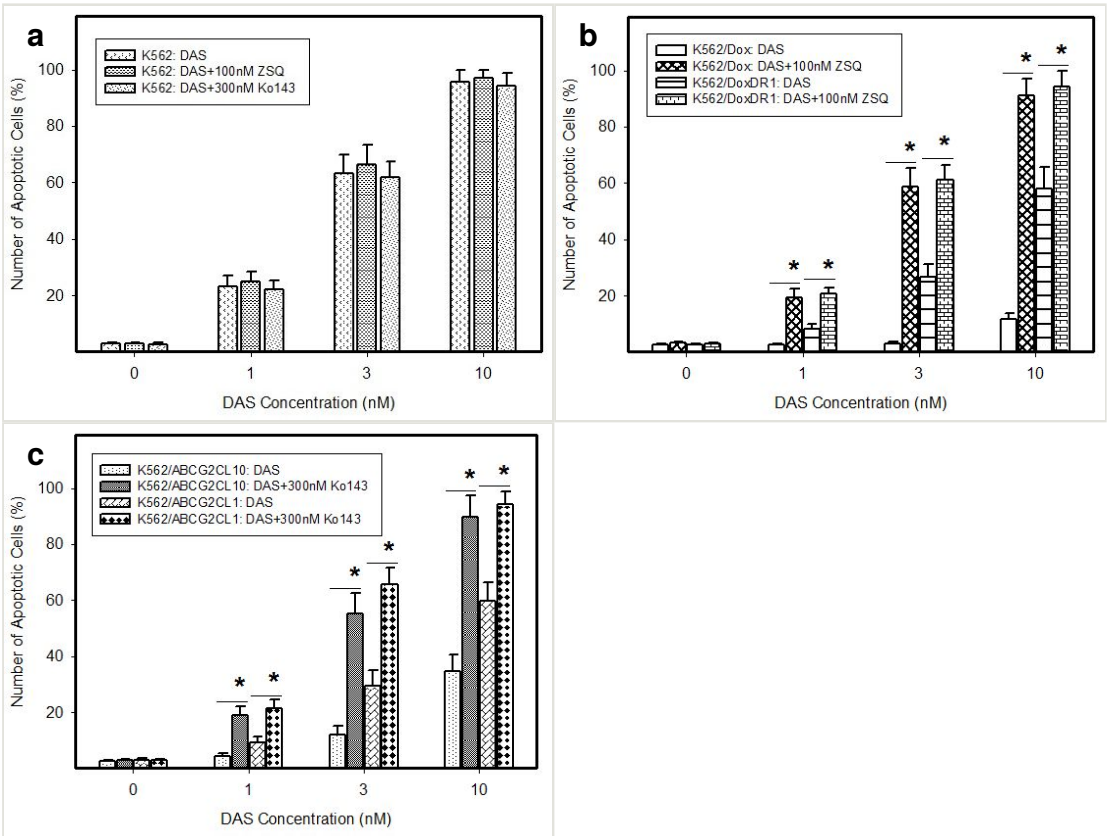

Figure 3.

Pro-apoptotic effects of DAS in cells expressing ABCB1 or ABCG2. Cells were treated with DAS or with DAS + appropriate inhibitor, as indicated. After $48 \mathrm{~h}$ the number of apoptotic cells was determined using fluorescence microscopy. The experimental points represent mean values from three replicate experiments, with standard deviations.

a: Induction of apoptosis by DAS treatment in parental cell line K562, which does not express any of transporters.

b: Induction of apoptosis by DAS treatment in cells overexpressing ABCB1 transporter. * denotes significant change in the number of apoptotic cells $(P<0.05)$ between resistant K562/ Dox and K562/DoxDR1 cells treated with DAS and DAS+ZSQ.

c: Induction of apoptosis by DAS treatment in cells overexpressing ABCG2 transporter. denotes significant change in the number of apoptotic cells $(P<0.05)$ between resistant ABCG2CL10 and ABCG2CL1 cells treated with DAS and DAS+Ko143. 


\section{Discussion}

MDR to chemotherapy is a serious obstacle in the treatment of cancer patients. Cancer MDR is defined as the cross-resistance or insensitivity of cancer cells to the cytostatic or cytotoxic actions of various anticancer drugs which are structurally or functionally unrelated and have different molecular targets (Gottesman 1993). Numerous mechanisms have been suggested to mediate MDR in cancer cells. In the last decades, a considerable attention has been paid to the role of $A B C$ transporters, and in particular to $A B C B 1, A B C C 1$, and ABCG2. In vitro studies on cultures of cancer cells usually consistently show a clear association between $A B C B 1, A B C C 1$, or $A B C G 2$ expression and resistance to chemotherapy. However, the correlation between the above mentioned $A B C$ transporters and clinical drug resistance is difficult to establish (Tamaki et al. 2011, Amiri-Kordestani et al. 2012).

Controversy in results is a common feature of studies evaluating the expression and prognostic role of these proteins and is mostly attributed to methodical factors, largely caused by an insufficient reliability and accuracy of methods used for the assessment of the $A B C$ transporters expression and function (Beck et al. 1996, Chevillard et al. 1997). Indeed, there is no doubt that assay methods for evaluation of $A B C$ transporters expression and function are important and may fundamentally affect the results. In addition, not always the assessment of ABCB1 expression can predict its function even in well-defined in vitro experiments (Kosztyu et al. 2015).

We believe that contradictory results might be due to oversimplification of evaluation of the MDR phenotype in cancer cells. The approach to this issue must be more complex. Therefore, if we want to evaluate a contribution of a particular $A B C$ transporter to the drug resistance, a clear quantitative relationship among intracellular drug level, particular $A B C$ transporter expression level, and cell sensitivity to this drug must be established. Importantly, the transporter expression level must be relevant to that found in clinical samples (Ambudkar et al. 2003).

The later requirement is crucial as the transporter expression level significantly affects the results. The higher transporter expression level effluxes drugs with increased efficiency and thus mediates higher resistance. While some drugs are effluxed effectively even in cells with low transporter expression levels, others are effluxed only poorly or their transport is below detection limits (Kosztyu et al. 2013, Kosztyu et al. 2014).

Similarly here, observed resistance to DAS depends on $A B C B 1$ and $A B C G 2$ expression levels (Figs 1, 2, 3, Table 1). Not surprisingly, K562/Dox and K562/ABCGCL10 cells expressing high levels of drug transporters, which are often used in laboratory experiments, exhibit a high degree of resistance to DAS (Figs 1, 2, 3, Table 1). However, K562/DoxDR1 and K562/ABCGCL1 cells with low expression levels of drug transporters, which likely can occur in clinical samples, exhibited much lower but significant degree of resistance to DAS (Figs 1, 2, 3, Table 1). In the context of our results, DAS appears to belong to the drugs which are effluxed effectively even in cells with low expression levels of 
$A B C B 1$ and $A B C G 2$. On the other hand, there may exist even lower expression levels of these drug transporters which will mediate lower degree of resistance to DAS. Alternatively, resistance may decrease below detection limits or may be lost.

Our results correspond to the finding of other authors who demonstrated that DAS is a substrate of both efflux proteins, $A B C B 1$ and ABCG2 and that these transporters could mediate resistance to this drug (Hegedűs et al. 2009, Hiwase et al. 2008, Dohse et al. 2010). However, these authors used cell lines with high expression levels of ABCB1 or ABCG2. These authors did not consider transporter expression level as important factor affecting results (Hegedűs et al. 2009, Hiwase et al. 2008, Dohse et al. 2010). There is one report by Hiwase and co-workers who demonstrated that neither ABCB1 nor ABCG2 affected intracellular levels of DAS in chronic myeloid leukemia-CD34+ (CML-CD34+) progenitors (Hiwase et al. 2013). Importantly, expression levels of drug transporters in CML-CD34+ was very low (Hiwase et al. 2013). These results indicate crucial importance of drug transporter expression levels for results interpretation.

We think that contradictory results that exist between in vitro experiments and clinics are mostly due to the different expression levels of drug transporters. Indeed, laboratory experiments usually relay on drug selected cell lines or transfected cells with enhanced expression of a studied transporter. Such cells always exhibit huge expression levels of the drug transporter which hardy occur in clinical samples. Data mined from The Cancer Genome Atlas (TCGA) indicates much higher expression levels of ABCB1 and ABCG2 in the normal tissue than in cancer counterpart pairs (Reinhold et al. 2015). We believe that our approach has a high potential to estimate real contribution of a particular $A B C$ transporter to the clinical drug resistance.

In this work we also address another important issue, namely the concentration of the studied drug in the experimental system used. Recent reports suggested that the interaction between TKIs and $A B C$ transporters is more complex. Indeed, some TKIs, including DAS, may serve as substrates of ABCB1 or ABCG2 at low concentrations, and as their inhibitors at high concentrations (Dohse et al. 2010, Eadie et al. 2014). For clinically relevant results it is necessary to use clinically relevant concentrations of TKIs. Concentrations of DAS in plasma were reported to be around 200nM (Eadie et al. 2014). Therefore, we used submicromolar concentrations of DAS in our study, 300nM DAS at the most (Figs 2, 3 and Table 1).

In conclusion, the antiproliferative and pro-apoptotic effects of DAS might be reduced by $A B C B 1$ or $A B C G 2$ overexpression at clinically relevant concentration. However, the actual effect of the studied $A B C$ transporters on DAS efficiency depends on their expression levels. The lower expression levels of $A B C$ transporters mediate lower resistance. Considering the fact that expression levels of ABCB1 and ABCG2 transporters are hardly high in clinical samples, their contribution to the overall resistance to DAS is probably low but significant. 


\section{Materials and methods}

\section{Chemicals}

Dasatinib monohydrate (DAS, Spyracel, BMS-354825), IUPAC name N-(2-chloro-6methylphenyl)-2-(6-(4-(2-hydroxyethyl)piperazin-1-yl)-2-methylpyrimidin-4ylamino)thiazole-5-carboxamide, was obtained from Selleckchem (Munich, Germany). Zosuquidar trihydrochloride (ZSQ; LY335979), IUPAC name $(\alpha R)-4-[(1 \mathrm{a} \alpha, 6 \alpha, 10 \mathrm{~b} \alpha)-1,1-$ difluoro-1,1a,6,10b-tetrahydrodibenzo[a,e]cyclopropa[c]cyclohepten-6-yl]- $\alpha-[(5-$

quinolinyloxy)methyl]-1-piperazineethanol trihydrochloride, was purchased from Selleckchem (Huston, TX, USA). Ko143 (3S,6S,12aS)-1,2,3,4,6,7,12,12a-Octahydro-9methoxy-6-(2-methylpropyl)-1,4-dioxopyrazino-[1',2':1,6] pyrido [3,4-b]indole-3-propanoic acid 1,1-dimethylethyl ester were obtained from Enzo Life Sciences AG (Lausen, Switzerland).

\section{Cell Culture}

Human chronic myelogenous leukemia K562 cells, obtained from ECACC, were cultured in the RPMI-1640 medium supplemented with a $10 \%$ calf foetal serum and antibiotics in $5 \%$ $\mathrm{CO}_{2}$ atmosphere at $37^{\circ} \mathrm{C}$. K562/Dox cells, which overexpress P-gp (ABCB1, MDR1), were kindly provided by Prof J.P. Marie (University of Paris 6, France). K562/Dox cells were cultured under the same conditions. More detailed characterisation of K562/Dox cell line is given elsewhere (Tang et al. 2008).

K562/DoxDR1 cells with down-regulated expression of P-gp were established by stable transfection of K562/Dox cells with a plasmid vector expressing shRNA targeting the ABCB1 gene (Mlejnek et al. 2012, Kosztyu et al. 2013).

K562/ABCG2 cells, which overexpress wild type ABCG2 (BCRP1), were kindly provided by Prof B. Sarkadi (National Blood Center and Semmelweis University, Budapest, Hungary). K562/ABCG2 cells were cultured under the same conditions. Detailed characterisation of K562/ABCG2 cell line is given elsewhere (Elkind 2005, Hegedűs et al. 2009).

Cells expressing different levels of ABCG2 were established by a single cell cloning by limiting dilution of K562/ABCG2 cells (Kosztyu et al. 2014). In this study we used two subclones, K562/ABCG2CL10 and K562/ABCG2CL1, with high and low expression level of $A B C G 2$, respectively. The detailed characteristics of the obtained subclones are given elsewhere (Kosztyu et al. 2014).

\section{$A B C B 1$ and $A B C G 2$ expression analysis using flow cytometry}

ABCB1 expression was studied by using UIC2 (Beckman Coulter, USA) monoclonal antibody conjugated with phycoerythrin (UIC2-PE) according to the manufacturer's instruction. Phycoerythrin conjugated isotype IgG2a was used as a control. ABCG2 expression was studied by using mouse anti-human CD 338 (BD Biosciences, USA) monoclonal antibody conjugated with phycoerythrin (CD338-PE) according to the 
manufacturer's instruction. Phycoerythrin conjugated isotype IgG2b was used as a control. The fluorescence of the cells was analysed by flow cytometry (Cytomics FC500, Beckman Coulter, USA). ABCB1 expression was determined by the ratio of the mean fluorescence intensity (MFI) shift of UIC2-PE antibody to isotype control (UIC2-PE/lgG2a-PE). Similarly, ABCG2 expression was determined by the ratio of the MFI shift of CD338-PE antibody to isotype control (CD338- PE/lgG2b-PE). For each sample 10000 events were collected. All the experiments were performed in triplicate.

\section{Determination of cell survival and proliferation}

The MTT assay was used for estimation of cell viability and growth as originally described by Mosmann (Mosmann 1983). Briefly, cells were seeded at concentration $7-8 \times 10^{4} \mathrm{cell} / \mathrm{s} / \mathrm{ml}$ and treated with DAS (or with DAS in combination with appropriate inhibitor) for $48 \mathrm{~h}$ under standard conditions at $37^{\circ} \mathrm{C}$. Untreated cells were used as control. Afterwards $0.5 \mathrm{mg} / \mathrm{ml}$ of MTT (final concentration) was added and incubation proceeded for $1 \mathrm{~h}$ at $37^{\circ} \mathrm{C}$. Cell pellets were extracted by isopropanol acidified with $0.01 \mathrm{MHCl}$. Absorbance was read at $570 \mathrm{~nm}$ in extracts clarified by centrifugation. The amount of formazane produced is proportional to the number of live and metabolically active cells.

\section{Morphological analysis of apoptosis}

Cells were fixed and stained with Hoechst 33342 (Fluka), as described previously (Mlejnek and Kuglik 2000). Nuclear morphology was examined by using an Olympus BX60 (Olympus, Hamburg, Germany) fluorescence microscope.

\section{Preparation of cell extracts}

The method is based on an optimised extraction of cells with formic acid after their separation from the growth medium by centrifugation through a layer of silicone oil (Mlejnek et al. 2011) with a slight modification. Briefly, cells at the density of $5 \times 10^{5} / \mathrm{ml}$ were incubated in the growth medium with $0,3 \mu \mathrm{M}$ DAS for $3 \mathrm{~h}$ in $5 \% \mathrm{CO}_{2}$ atmosphere at $37^{\circ} \mathrm{C}$. The cells were then centrifuged through silicone oil and cell pellets were extracted using ice cold $1 \%(\mathrm{w} / \mathrm{v})$ formic acid $+50 \%(\mathrm{v} / \mathrm{v})$ methanol in water. Cell extracts were clarified by centrifugation $\left(40000 \mathrm{~g} \mathrm{x} 10 \mathrm{~min}\right.$ at $4^{\circ} \mathrm{C}$ ), diluted with extraction solution and analysed by liquid chromatography coupled with a low-energy collision tandem mass spectrometer (LC/ MS/MS).

\section{Assay for determination of intracellular DAS levels}

The HPLC system consisted of UltiMate 3000 RS pump, degasser, autosampler and column compartment (Dionex, Germering, Germany). Separations were performed at ambient temperature on a Polaris C18-A $150 \times 2.0 \mathrm{~mm}$ (i.d.), $5 \mu \mathrm{m}$ particle size column (Varian Inc., Lake Forest, CA, USA) connected with a guard C18 $4.0 \times 2.0 \mathrm{~mm}$ (i.d.) precolumn (Phenomenex, Torrance, CA, USA). Solvents used for separation were A (95\% methanol in $0.25 \% \mathrm{FA}, \mathrm{v} / \mathrm{v})$ and $\mathrm{B}(0.5 \% \mathrm{FA}, \mathrm{v} / \mathrm{v})$. The flow rate was $250 \mu \mathrm{l} / \mathrm{min}$ with linear 
gradient elution from 0 to $3 \mathrm{~min}$ (60 to $95 \%$ of solvent $A$ ), from 3 to $4 \mathrm{~min}$ ( $95 \%$ of solvent A), from 4 to $5 \mathrm{~min}$ (95 to $60 \%$ of solvent A) and from 5 to $8 \mathrm{~min}(60 \%$ of solvent $A$ ). Sample injection volume was set at $10 \mu \mathrm{l}$. The effluent was introduced into the API 3200 triple quadrupole mass spectrometer (MDS SCIEX, Ontario, Canada) and electrospray ionization in positive ion mode was used for detection. The mass spectrometer was operated in the multiple-reaction monitoring (MRM) mode. DAS was monitored by MRM transition $488>401$ (dwell-time $=150$ miliseconds). lon spray probe parameters were set to the following values: needle voltage $5500 \mathrm{~V}$, temperature $400^{\circ} \mathrm{C}$, curtain gas (nitrogen) 1.38bar, nebulizer gas (zero air quality) 3.45 bar, turbo V-gas (zero air quality) 3.45 bar. The nitrogen pressure in the second quadrupole was measured at $4.0 \times 10^{-8}$ bar. The declustering potential, the collision energy and the entrance potential were set at $61 \mathrm{~V}, 33 \mathrm{~V}$ and $7.5 \mathrm{~V}$, respectively. The instrument was operated in unit resolution. Data were collected and processed using Analyst ${ }^{\circledR}$ software (version 1.5.1).

\section{Statistical analysis}

Data are reported as the mean \pm S.D. Statistical significance of differences was determined by Student's t-test. Only the $P$ values less than 0.05 were considered significant.

\section{Acknowledgements}

This work was supported by grant No: IGA_LF_2016_035 (Internal grant of Palacky University).

\section{Conflicts of interest}

Authors declare that they are not in any conflict of interest.

\section{References}

- $\quad$ Ambudkar SV, Kimchi-Sarfaty C, Sauna ZE, Gottesman MM (2003) P-glycoprotein: from genomics to mechanism. Oncogene 22 (47): 7468-7485. https://doi.org/10.1038/ sj.onc. 1206948

- $\quad$ Amiri-Kordestani L, Basseville A, Kurdziel K, Fojo AT, Bates S (2012) Targeting MDR in breast and lung cancer: Discriminating its potential importance from the failure of drug resistance reversal studies. Drug Resistance Updates 15: 50-61. https:// doi.org/10.1016/i.drup.2012.02.002

- $\quad$ Beck WT, Grogan TM, Willman CL, Cordon-Cardo C, Parham DM, Kuttesch JF, Andreeff M, Bates SE, Berard CW, Boyett JM, Brophy NA, Broxterman HJ, Chan HS, Dalton WS, Dietel M, Fojo AT, Gascoyne RD, Head D, Houghton PJ, Srivastava DK, Lehnert M, Leith CP, Paietta E, Pavelic ZP, Weinstein R (1996) Methods to detect P- 
glycoprotein-associated multidrug resistance in patients' tumors: consensus recommendations. Cancer research 56 (13): 3010-20.

- Chevillard S, Vielh P, Validire P, Marie JP, Faussat AM, Barbu V, Bayle C, Bénard J, Bonnal C, Boutonnat J, Calvo F, Charrier J, Clary A, Colosetti P, Danel-Moore L, Decrémoux P, Delvincourt C, Finat-Duclos F, Genne P, Kataki A, Kouyoumdjian JC, Lacave R, Maugard C, Merlin JL, Mousseau M, Pinguet F, Quillien V, Raphael M, Richard B, Verrelle P, Robert J (1997) French multicentric evaluation of mdr1 gene expression by RT-PCR in leukemia and solid tumours. Standardization of RT-PCR and preliminary comparisons between RT-PCR and immunohistochemistry in solid tumours. Leukemia 11 (7): 1095-1106. https://doi.org/10.1038/sj.leu.2400657

- Dohse M, Scharenberg C, Shukla S, Robey RW, Volkmann T, Deeken JF, Brendel C, Ambudkar SV, Neubauer A, Bates SE (2010) Comparison of ATP-Binding Cassette Transporter Interactions with the Tyrosine Kinase Inhibitors Imatinib, Nilotinib, and Dasatinib. Drug Metabolism and Disposition 38 (8): 1371-1380. https://doi.org/10.1124/ dmd.109.031302

- Dy G, Adjei A (2008) Systemic cancer therapy: Evolution over the last 60 years. Cancer 113: 1857-1887. https://doi.org/10.1002/cncr.23651

- $\quad$ Eadie LN, Hughes TP, White DL (2014) Interaction of the efflux transporters ABCB1 and ABCG2 with imatinib, nilotinib, and dasatinib. Clinical pharmacology and therapeutics 95 (3): 294-306. https://doi.org/10.1038/clpt.2013.208

- $\quad$ Elkind NB (2005) Multidrug Transporter ABCG2 Prevents Tumor Cell Death Induced by the Epidermal Growth Factor Receptor Inhibitor Iressa (ZD1839, Gefitinib). Cancer Research 65 (5): 1770-1777. https://doi.org/10.1158/0008-5472.can-04-3303

- Gottesman MM (1993) How cancer cells evade chemotherapy: sixteenth Richard and Hinda Rosenthal Foundation Award Lecture. Cancer research 53 (4): 747-54.

- Hegedűs C, Özvegy-Laczka C, Apáti Á, Magócsi M, Német K, Örfi L, Kéri G, Katona M, Takáts Z, Váradi A, Szakács G, Sarkadi B (2009) Interaction of nilotinib, dasatinib and bosutinib with $A B C B 1$ and $A B C G 2$ : implications for altered anti-cancer effects and pharmacological properties. British Journal of Pharmacology 158 (4): 1153-1164. https://doi.org/10.1111/j.1476-5381.2009.00383.x

- Hiwase DK, Saunders VA, Nievergall E, Ross DD, White DL, Hughes TP (2013) Dasatinib targets chronic myeloid leukemia-CD34+ progenitors as effectively as it targets mature cells. Haematologica 98 (6): 896-900. https://doi.org/10.3324/ haematol.2012.070268

- $\quad$ Hiwase DK, Saunders V, Hewett D, Frede A, Zrim S, Dang P, Eadie L, To LB, Melo J, Kumar S, Hughes TP, White DL (2008) Dasatinib Cellular Uptake and Efflux in Chronic Myeloid Leukemia Cells: Therapeutic Implications. Clinical Cancer Research 14 (12): 3881-3888. https://doi.org/10.1158/1078-0432.ccr-07-5095

- Holló Z, Homolya L, Davis CW, Sarkadi B (1994) Calcein accumulation as a fluorometric functional assay of the multidrug transporter. Biochimica et Biophysica Acta (BBA) Biomembranes 1191 (2): 384-388. https://doi.org/10.1016/0005-2736(94)90190-2

- Kantarjian HM, Giles F, Quintas-Cardama A, Cortes J (2007) Important Therapeutic Targets in Chronic Myelogenous Leukemia. Clinical Cancer Research 13 (4): 1089-1097. https://doi.org/10.1158/1078-0432.ccr-06-2147

- Kosztyu P, Dolezel P, Mlejnek P (2013) Can P-glycoprotein mediate resistance to nilotinib in human leukaemia cells? Pharmacological Research 67 (1): 79-83. https:// doi.org/10.1016/j.phrs.2012.10.012 
- Kosztyu P, Bukvova R, Dolezel P, Mlejnek P (2014) Resistance to daunorubicin, imatinib, or nilotinib depends on expression levels of $A B C B 1$ and $A B C G 2$ in human leukemia cells. Chemico-Biological Interactions 219: 203-210. https://doi.org/10.1016/ j.cbi.2014.06.009

- $\quad$ Kosztyu P, Dolezel P, Vaclavikova R, Mlejnek P (2015) Can the assessment ofABCB1gene expression predict its function in vitro? European Journal of Haematology 95 (2): 150-159. https://doi.org/10.1111/ejh.12470

- Mlejnek P, Kuglik P (2000) Induction of apoptosis in HL-60 cells by N6-benzyladenosine. Journal of Cellular Biochemistry 77 (1): 6-17. https://doi.org/10.1002/(sici)1097-4644 (20000401)77:13.0.co;2-3

- $\quad$ Mlejnek P, Dolezel P, Kosztyu P (2012) P-glycoprotein mediates resistance to A3 adenosine receptor agonist 2-chloro-N6-(3-iodobenzyl)-adenosine-5'-nmethyluronamide in human leukemia cells. Journal of cellular physiology 227 (2): 676-85. https://doi.org/10.1002/jcp.22775

- Mlejnek P, Novak O, Dolezel P (2011) A non-radioactive assay for precise determination of intracellular levels of imatinib and its main metabolite in Bcr-Abl positive cells. Talanta 83 (5): 1466-1471. https://doi.org/10.1016/j.talanta.2010.11.028

- Mosmann T (1983) Rapid colorimetric assay for cellular growth and survival: application to proliferation and cytotoxicity assays. Journal of immunological methods 65 : 55-63. https://doi.org/10.1016/0022-1759(83)90303-4

- $\quad$ Nam S, Williams A, Vultur A, List A, Bhalla K, Smith D, Lee FY, Jove R (2007) Dasatinib (BMS-354825) inhibits Stat5 signaling associated with apoptosis in chronic myelogenous leukemia cells. Molecular Cancer Therapeutics 6 (4): 1400-1405. https:// doi.org/10.1158/1535-7163.mct-06-0446

- $\quad$ Reinhold WC, Sunshine M, Varma S, Doroshow JH, Pommier Y (2015) Using CellMiner 1.6 for Systems Pharmacology and Genomic Analysis of the NCl-60. Clinical Cancer Research 21 (17): 3841-3852. https://doi.org/10.1158/1078-0432.ccr-15-0335

- Robey RW, Steadman K, Polgar O, Morisaki K, Blayney M, Mistry P, Bates SE (2004) Pheophorbide a Is a Specific Probe for ABCG2 Function and Inhibition. Cancer Research 64 (4): 1242-1246. https://doi.org/10.1158/0008-5472.can-03-3298

- Tamaki A, lerano C, Szakacs G, Robey R, Bates S (2011) The controversial role of ABC transporters in clinical oncology. Essays In Biochemistry 50: 209-232. https:// doi.org/10.1042/bse0500209

- $\quad$ Tang R, Faussat A, Perrot J, Marjanovic Z, Cohen S, Storme T, Morjani H, Legrand O, Marie J (2008) Zosuquidar restores drug sensitivity in P-glycoprotein expressing acute myeloid leukemia (AML). BMC Cancer 8 (1). https://doi.org/10.1186/1471-2407-8-51 